\title{
ANÁLISE DAS BOAS PRÁTICAS DE MANEJO NA CARCINICULTURA E SEUS EFEITOS SOBRE A PRODUTIVIDADE NO ESTADO DO CEARÁ
}

\section{Analysis of the good management practices in the shrimp farming and its effects on the productivity in Ceará}

\author{
Ana Maria Maurício Araújoํㅜㄹ Rogério César Pereira de Araújo², \\ Rosemeiry Melo Carvalho ${ }^{3}$, Lucas David Ribeiro Reis ${ }^{4}$
}

${ }^{1}$ B.S. em Engenharia de Pesca e M.S. em Desenvolvimento e Meio Ambiente. Universidade Federal do Ceará - UFC. Av. Mister Hull, 2977, Campus do Pici, CEP 60021-970, Bloco 826, Fortaleza, CE, Brasil.anamm_a@yahoo.com.br

${ }^{2}$ Professor do Departamento de Economia Agrícola da Universidade Federal do Ceará (UFC). B.S. em Engenharia Agronômica, M.S. em Economia Rural, e Ph.D. em Economia Agrícola. Departamento de Economia Agrícola/UFC, CP 6017, Campus do Pici, Fortaleza-CE, CEP 60.455-970, Brasil. rcpa@ufc.br

${ }^{3}$ Professora do Departamento de Economia Agrícola da Universidade Federal do Ceará (UFC). B.S. em Engenharia Agronômica, M.S. em Economia Rural, Dra. Em Economia. Departamento de Economia Agrícola/UFC, CP 6017, Campus do Pici, Fortaleza-CE, CEP 60.455-970, Brasil. rmelo@ufc.br

${ }^{4}$ Estudo do Mestrado em Economia Rural da Universidade Federal do Ceará (UFC). Departamento de Economia Agrícola/UFC, CP 6017, Campus do Pici, Fortaleza-CE, CEP 60.455-970, Brasil. econ.lucasdavid@gmail.com

\section{RESUMO}

A eficiência técnica na carcinicultura depende cada vez mais da qualidade dos insumos empregados na produção e das práticas de manejo realizado na fazenda. Esta pesquisa teve como objetivo descrever as boas práticas, produtivas e ambientais, adotadas pela carcinicultura, no estado do Ceará, e avaliar a relação entre essas práticas e a produtividade de camarão cultivado neste estado. Para efetuar os testes de significância das práticas adotadas, uma amostra de 60 fazendas de engorda de camarão foi coletada e utilizada para estimar um modelo de regressão semi-logarítmica. Os resultados mostraram que as fazendas amostradas possuíam nível médio de adoção das boas práticas e que aquelas voltadas para a melhoria da qualidade ambiental na fazenda não afetaram significativamente a produtividade média, evidenciando que a busca da eficiência técnica produtiva tem sido a prioridade nas fazendas.

Palavras-chave: Camarão, Sustentabilidade, Eficiência técnica.

Recebido em: 01/10/2017

Aprovado em: 23/06/2018

Publicado online em: 05/09/2018 


\section{ABSTRACT}

The technical efficiency of the shrimp farming increasingly depends on the quality of inputs used in the production and management practices carried out in the farm. This research aimed to describe the good practices, productive and environmental, adopted by shrimp farms, in the State of Ceará, and assess the relationship among the those practices and shrimp farming productivity in this State. To do the significance tests of the adopted practices, a sample of 60termination shrimp farms was gathered and used to estimate a semi-logarithm regression model. The results showed that the farms sampled had mean level of adopting the good practices and that those directed to improve of the environmental quality in the farm did not affected significantly the mean productivity, which revealed that targeting the productive technical efficiency has been the priority in the farms.

Key words: Shrimp, Sustainability, Technical efficiency.

\section{INTRODUÇÃO}

No ano de 2015, o Estado do Ceará assumiu a posição de maior produtor de camarão cultivado brasileiro, com 41.414 toneladas, o que correspondia a 58,1\% da produção nordestina e $57,7 \%$ da produção nacional. Essa produção foi obtida a partir de uma área de 41.414 ha, distribuídas entre 630 produtores (IBGE, 2015). No ano seguinte, embora o número de produtores e área tenha aumentado para 700 e 10.407 hectares, respectivamente, a produção de camarão declinou em 38,6\%, passando para 25.431 toneladas (IBGE, 2016).

Apesar disso, a produtividade média, no Ceará, tem experimentado um declínio acentuado. Entre 2003 e 2016, a produtividade regrediu 60\%, passando de 6.084 para $2.443,68 \mathrm{~kg} / \mathrm{ha} /$ ano (Tahim \& Araújo Junior, 2014). Apenas no ano de 2016, relativo ao ano anterior, a produtividade média passou de 4.250 para $2.444 \mathrm{~kg} / \mathrm{ha} / \mathrm{ano}$, correspondendo a um declínio de 42,5\% (IBGE, 2016).

O declínio da produtividade do camarão cultivado deve-se, em parte, à disseminação do vírus da mionecrose infecciosa (IMNV), que afetou adversamente os cultivos no estado, a partir de 2004 (ABCC, 2017). Em 2016, o vírus da mancha branca (WSSV) alcançou os cultivos de camarão cearenses, o que vem causando elevada mortalidade (ABCC, 2017). Associa-se o surgimento dessas doenças à precariedade do manejo produtivo e ambiental das carciniculturas.

Neste sentido, o debate baseado na gestão ambiental tem focado nos fatores determinantes da eficiência técnica da produção de camarão marinho cultivado no Brasil. Cada vez mais, ganhos de eficiência na produção são obtidos por meio da melhoria da qualidade ambiental dentro da fazenda e no seu entorno (Ormond et al., 2004).

Alguns estudos mostram como a produtividade por unidade de área de viveiro pode ser aumentada por meio da adoção de práticas de manejo na fazenda. Por exemplo, a competitividade da carcinicultura pode ser aumentada quando se elimina o desperdício dos insumos, faz o reuso dos resíduos da produção e reduz os custos de produção (Feitosa, 2005). Jory (1995 apud Souza Júnior, 2003) ressaltou que o complexo manejo dos viveiros requer o conhecimento sobre a biologia das espécies cultivadas, a qualidade da água e do solo, e o contínuo monitoramento da produção. 
Neste contexto, a distribuição do alimento natural é o principal componente do manejo dos viveiros e fator crítico para a produção eficiente. ${ }^{5} \mathrm{O}$ uso de alta densidade de pós-larvas tem sido apontado como o principal responsável pela obtenção de produtividade elevada, porém, esta prática necessita de cuidado especial, no que diz respeito à questão da sanidade dos cultivos (Fonseca, 2005).

Valenti (2002) destaca a importância do uso da água de despesca para o aumento da produtividade, visto que o referido sistema aproveita melhor os nutrientes (Alencar, Horta Júnior \& Celino, 2010). Waite et al. (2001) defendem que o aumento da produtividade da carcinicultura pode ser alcançada por meio da "intensificação sustentável", que se traduz por meio da promoção do desenvolvimento socioeconômico, uso de alimentos seguros e nutritivos, e minimização da poluição da água.

No Brasil, a adoção das boas práticas de manejo é ainda bastante desigual entre os carcinicultores. Por exemplo, comedouros fixos são adotados por $88 \%$ dos produtores, enquanto os berçários intensivos são empregados por apenas $12 \%$. Quanto ao emprego de probióticos, os produtores de tamanho médio/grande fazem uso dessas substâncias com maior frequência do que os de tamanho micro/pequeno (Rodrigues \& Borba, 2013).

Com base no exposto, pode-se afirmar que, no estado do Ceará, existe margem para aumentar a produtividade da carcinicultura através da intensificação da adoção das práticas de manejo produtivo e/ou ambiental na fazenda. Neste sentido, torna-se necessário caracterizar o nível tecnológico atual das fazendas e seu efeito sobre a produtividade. Assim, este estudo tem como objetivo investigar se a eficiência técnica produtiva, em particular a produtividade por unidade de área cultivada, está associada positivamente às boas práticas de manejo dos cultivos.

Para entender melhor esta relação, fez-se necessário, primeiramente, a caracterização das práticas de manejo produtivo e ambiental na carcinicultura cearense. Em seguida, com base nas práticas adotadas nas fazendas, foram estimados indicadores de manejo e um índice de adoção das boas práticas de manejo para as fazendas. Por último foi estimado um modelo econométrico para avaliar a relação empírica entre a produtividade média por unidade de área cultivada das fazendas e as práticas de manejo produtivo e ambiental adotadas na fazenda.

O artigo é composto por cinco seções, incluindo esta primeira, na qual foram definidos o problema e os objetivos do estudo. Na segunda seção foi feita uma breve exposição do arcabouço institucional relacionado à gestão ambiental na carcinicultura no Brasil. Na terceira seção foi apresentada a metodologia da pesquisa, no qual restou delimitada a área de estudo, além da especificação do modelo de regressão e definidas as variáveis a serem utilizadas na análise. Na quarta seção foram analisados os resultados dos modelos e, finalmente, na quinta seção foram apresentadas as conclusões do estudo.

\section{GESTÃO AMBIENTAL NA CARCINICULTURA}

De forma geral, a gestão ambiental é um instrumento de planejamento que incorpora os princípios do desenvolvimento sustentável direcionados à organização social e metas corporativas da empresa (Moreno \& Pol, 1999). Dentre esses princípios, destaca-se a neces-

5 O manejo ótimo do alimento resulta em taxas máximas de crescimento e sobrevivência e menor conversão alimentar com fornecimento de ração e impacto ambiental, mínimos. 
sidade de integrar as políticas, programas e práticas relativas ao meio ambiente em um processo contínuo de melhoria da gestão e a redução da geração de resíduos e minimização dos riscos ambientais. Assim, as empresas, além de se preocuparem com os resultados financeiros, devem incorporar também a questão da justiça social e a qualidade ambiental, ou seja, os resultados devem ser analisados em três dimensões (triple bottom line) (Silva Filho, 2008).

Essa nova concepção de gestão passou a exigir da empresa mudança de postura, que se traduziu na obediência às leis, busca de eficácia nos custos, respeito à opinião pública e planejamento de longo prazo. Assim, além de proteger o meio ambiente, a gestão ambiental tornou-se também uma fonte de lucro para os negócios, tendo em vista que empresas que respeitam o meio ambiente terão maior notoriedade diante dos órgãos de fiscalização, consumidores e credores.

Na carcinicultura, a gestão ambiental tem sido orientada pelas leis, regulamentações, códigos de conduta e os guias de boas práticas de gestão ambiental (Feitosa, 2005). No Brasil, a carcinicultura é regida pela Lei $n^{0} 7.661 / 1988$ e Resolução $n^{0} 312 / 2002$ do CONAMA (Brasil, 2002). Os princípios de gestão ambiental para a carcinicultura estão definidos nos seguintes guias: Global Aquaculture Alliance (GAA); Código de Conduta e o Guia de Boas Práticas de Manejo e de Fabricação para uma Carcinicultura Ambientalmente Sustentável e Socialmente Justa (ABCC, 2005) e; Princípios para Boas Práticas de Manejo (BPM) na Engorda de Camarão Marinho no Estado do Ceará (Nunes et al., 2005).

Os estudos sobre a gestão ambiental em empreendimentos de carcinicultura no Brasil são ainda incipientes. Tahim (2006) verificou que as empresas de camarão cultivado, principalmente as médias e grandes empresas, adotam medidas somente para atender as exigências da legislação. Silva Filho et al. (2008), ao analisarem a conduta ambiental e social de empresas de carcinicultura do litoral do Estado do Ceará, verificaram que a maioria das empresas pesquisadas mantém um perfil de conduta fraca, tanto ambiental quanto social, sendo encontrada uma exceção.

Ainda segundo Silva Filho et al. (2008), o número de fazendas de carcinicultura que adotam medidas que visam proteger o meio ambiente ainda é bastante reduzida. Joventino (2008) enfatizou que, no estado do Ceará, a maioria dos empreendimentos estão localizados em áreas de preservação permanentes, ou seja, próximos às margens de rios e manguezais.

Desta forma, a gestão ambiental é um instrumento importante para a promoção do desenvolvimento sustentável da carcinicultura, haja vista a existência de um arcabouço legal já instituído e a informação disponível para orientar a gestão ambiental na carcinicultura. Porém, evidências apontam para baixa efetividade desta abordagem para a melhoria da qualidade ambiental em virtude do baixo grau de adoção pela grande maioria das fazendas.

\section{MATERIAL E MÉTODO}

\section{Dados}

A população da pesquisa é formada pelas fazendas de engorda do camarão marinho cultivados, localizadas no Ceará, abrangendo empreendimentos de pequeno, médio e grande porte. A amostra foi coletada pelo método de amostragem não probabilístico, por não se ter tido acesso à listagem completa de fazendas em operação na área de estudo. 
A amostra é formada por 60 fazendas de carcinicultura, sendo 41 (ou 68,3\%) delas localizadas no litoral leste e 19 (31,7\%), no litoral oeste. As fazendas selecionadas foram indicadas por técnicos que atuam na área de estudo. A Tabela 1 mostra a distribuição de frequências das fazendas por municípios.

\begin{tabular}{|c|c|c|}
\hline Macrorregião/Município & $\begin{array}{l}\text { Frequência } \\
\text { Absoluta } \\
\text { (N.) }\end{array}$ & $\begin{array}{c}\text { Frequência } \\
\text { Relativa } \\
\text { (\%) }\end{array}$ \\
\hline \multicolumn{3}{|l|}{ Litoral Leste: } \\
\hline Aracati & 18 & 30,0 \\
\hline Beberibe & 1 & 1,7 \\
\hline Fortim & 8 & 13,3 \\
\hline Icapuí & 1 & 1,7 \\
\hline Itaiçaba & 3 & 5,0 \\
\hline Jaguaruana & 9 & 15,0 \\
\hline Russas & 1 & 1,7 \\
\hline Subtotal (1) & 41 & 68,3 \\
\hline \multicolumn{3}{|l|}{ Litoral Oeste: } \\
\hline Acaraú & 9 & 15,0 \\
\hline Amontada & 2 & 3,3 \\
\hline Camocim & 2 & 3,3 \\
\hline Itarema & 2 & 3,3 \\
\hline Paracuru & 1 & 1,7 \\
\hline Paraipaba & 1 & 1,7 \\
\hline Trairi & 1 & 1,7 \\
\hline Itapipoca & 1 & 1,7 \\
\hline Subtotal (2) & 19 & 31,7 \\
\hline Total $(1+2)$ & 60 & 100,0 \\
\hline
\end{tabular}

Fonte: Elaboração dos autores.

Os municípios com maior frequência de fazendas na amostra foram: Aracati (30\%), Fortim (13,3\%) e Jaguaruana (15\%), no litoral leste e; Acaraú (15\%), no litoral oeste. O município de Acaraú, juntamente com os municípios de Jijoca de Jericoacoara, Cruz e Itarema compõem o Polo de carcinicultura do litoral oeste, denominado de Costa Negra, que abrange um total e 32 produtores da região.

Em relação à distribuição dos produtores ativos por município no estado do Ceará, o município de Aracati assumia a primeira posição em número de produtores, área cultivada e quantidade produzida. Em seguida, aparecem Acaraú, Camocim, Jaguaruana, Beberibe e Fortim como principais produtores, todos com produção individual superior a 1.000 toneladas anuais (Rodrigues \& Borba, 2013).

Em sua maioria, esses produtores adotam o sistema semi-intensivo de produção, o qual se caracteriza pelo uso intensivo de insumos, alta densidade de estocagem e manejo de elevado volume de efluentes, sendo considerada uma atividade de elevado potencial poluidor.

O questionário foi aplicado pessoalmente pela pesquisadora, na própria fazenda ou fora dela, em diversos locais, tais como: sede da empresa, sede da associação, eventos técnico-científicos, etc. A pesquisa foi realizada durante o ano de 2014.

\section{Método}

Esta análise tem como fundamento a função de produção da firma que descreve a relação entre o produto obtido pelo emprego de um conjunto de fatores de produção (in- 
sumos) por unidade de tempo. Asuming-Brempong (2010) utilizou a seguinte equação para representar uma função de produção em sua forma genérica:

$Q=f(X, Z) e^{u}$

onde:

$Q$ : produto obtido em uma atividade agrícola;

$X$ : vetor de insumos empregados na produção;

$Z$ : vetor de práticas adotadas na unidade produtiva;

$u$ : termo do erro aleatório e;

$e$ : número natural $(2,71828)$.

A produtividade média de um fator variável é uma medida de eficiência técnica amplamente utilizada para avaliar o desempenho de uma atividade produtiva. A vantagem desta medida está associada à sua simplicidade de cálculo e o fácil entendimento por parte do tomador de decisão.

A função de produtividade média de qualquer fator de produção $\left(X_{i}\right)$ é obtida a partir da equação (2), dividindo o produto total pela quantidade do fator, como mostrada a seguir:

$Q x_{i}=\frac{Q}{X_{i}}=g(X, Z) e^{u}$

onde:

$Q x_{i}:$ produtividade média do fator $i$;

$Q$ : produto total e;

$X_{i}$ : quantidade empregada do fator $i$.

A produtividade média do fator é uma variável aleatória, assim como o produto total $(Q)$. Desta forma, a função de produtividade média do fator é uma transformação monotônica da função de produção, ambas podendo ser explicadas pelo mesmo conjunto de variáveis.

Considerando apenas os efeitos aditivos diretos das variáveis explicativas sobre a produtividade média, a função de produtividade média assume a forma linear, como expressa pela equação:

$Q x_{i j}=\beta_{0}+\beta_{1} X_{1 j}+\ldots+\beta_{i} X_{i j}+\ldots+\beta_{n} X_{n j}+u_{j}$

onde:

$Q$ : a produtividade média da fazenda $j$; sendo $j=1, \ldots, \mathrm{J}$;

$\beta_{0}$ : o termo constante do modelo;

$\beta_{i}$ : o coeficiente da variável explicativa $X_{i j}$, sendo $i=1, \ldots, \mathrm{N}$;

$X_{i j}$ : a quantidade do fator $i$ da fazenda $j$; e

$u_{j}$ : o termo do erro aleatório da fazenda $j$.

Nesse modelo, a direção e a magnitude do efeito marginal de uma variável explicativa sobre a produtividade média são expressas pelo sinal e valor do coeficiente estimado, respectivamente. A produtividade média irá aumentar quando o coeficiente da variável for positivo e vice-versa. 
A variabilidade das variáveis não observadas, ou seja, aquelas não incluídas no modelo são capturadas pelo termo do erro aleatório. Tendo em vista que a regressão linear é estimada pelo método dos Mínimos Quadrados Ordinários (MQO), o erro aleatório tem distribuição normal com média igual a zero e desvio padrão constante $\left(\sigma^{2}\right)$.

As variáveis explicativas são distribuídas em três grupos, a saber: (i) características e localização da fazenda; (ii) características do sistema de produção; e (iii) indicadores e índice que descrevem as boas práticas adotadas na fazenda. As variáveis explicativas são, em sua maioria, nominais, estas sendo definidas como variáveis dummies, ou seja, a variável assume valor 1, quando a característica está presente na fazenda e valor 0 quando está ausente. A Tabela 2 apresenta a lista de variáveis do modelo, seguida de sua definição e decodificação.

Tabela 2 - Variáveis do modelo de produtividade média da área cultivada com relação a adoção de práticas de gestão ambiental da carcinicultura do Ceará.

\begin{tabular}{|c|c|c|}
\hline Variável & Definição & Código \\
\hline$P M E$ & Produtividade Média & Contínua \\
\hline AREAFAZ & Área da fazenda & Contínua \\
\hline AREAVIV & Área de viveiros & Contínua \\
\hline DENEST & Densidade de Estocagem & Contínua \\
\hline CVM & Custo Variável Médio & Contínua \\
\hline APISAL & $\begin{array}{l}\text { Tipo de solo onde se localiza a fazenda: apicum, salgado ou } \\
\text { tabuleiro. }\end{array}$ & $\begin{array}{l}\text { Binária: } 1 \text { se for apicum ou } \\
\text { salgado; } 0 \text { se tabuleiro ou outros }\end{array}$ \\
\hline RIOGAMB & $\begin{array}{l}\text { Fonte de água de abastecimento dos viveiros: rio, gamboa, estuário } \\
\text { ou outras fontes. }\end{array}$ & $\begin{array}{l}\text { Binária: } 1 \text { se for rio/gamboa; } 0 \text {, } \\
\text { se estuário ou outros }\end{array}$ \\
\hline TECGEN & $\begin{array}{l}\text { Tipo de assistência técnica permanente (engenheiro, técnico ou } \\
\text { gestor ambiental) ou periódica (consultoria e outras formas) }\end{array}$ & $\begin{array}{l}\text { Binário: } 1 \text { se permanente; } 0 \text {, se } \\
\text { periódica }\end{array}$ \\
\hline$I M V$ & $\begin{array}{l}\text { Indicador de Manejo dos Viveiros: Indicador que reflete a adoção de } \\
\text { práticas relacionadas ao manejo químico, físico e biológico dos } \\
\text { viveiros ativos. }\end{array}$ & Varia no intervalo de 0 a 1 \\
\hline$I M E$ & $\begin{array}{l}\text { Indicador de manejo de efluentes: Indicador que reflete a adoção de } \\
\text { práticas relacionadas à forma como os efluentes são gerados e } \\
\text { descartados na fazenda. }\end{array}$ & Varia no intervalo de 0 a 1 \\
\hline IMS & $\begin{array}{l}\text { Indicador de manejo de sedimentos: Indicador que reflete a adoção } \\
\text { de práticas relacionadas ao manejo de sedimentos na fazenda. }\end{array}$ & Varia no intervalo de 0 a 1 \\
\hline$I M D$ & $\begin{array}{l}\text { Indicador de manejo de despesca: Indicador que reflete a adoção de } \\
\text { práticas relacionadas ao manejo de metabissulfito de sódio antes e } \\
\text { durante a despesca. }\end{array}$ & Varia no intervalo de 0 a 1 \\
\hline$I C A$ & $\begin{array}{l}\text { Indicador de conservação ambiental: Indicador que reflete a adoção } \\
\text { de práticas de conservação e manutenção dos ecossistemas de } \\
\text { entorno à fazenda. }\end{array}$ & Varia no intervalo de 0 a 1 \\
\hline IGAPM & $\begin{array}{l}\text { Índice global de adoção de práticas de manejo: índice composto que } \\
\text { reflete a média dos indicadores de adoção de práticas de gestão } \\
\text { ambiental em cada fazenda. }\end{array}$ & Varia no intervalo de $0 \%$ a $100 \%$ \\
\hline
\end{tabular}

Fonte: Elaboração dos autores.

As medidas de Área da Fazenda e Área de Viveiros são definidas, respectivamente, como a área total da propriedade e a área efetiva com espelho d’água, em hectares.

A Densidade de Estocagem corresponde ao número de indivíduos estocados em uma determinada área $\left(\mathrm{cam} . / \mathrm{m}^{2}\right)$. Esta variável está diretamente relacionada ao tipo de sistema de produção, sendo assim classificado: extensiva, densidade de estocagem menor que 2,5 cam./ $\mathrm{m}^{2}$; semi-intensivo, entre 2,5 e $20 \mathrm{cam} . / \mathrm{m}^{2}$; e intensivo, maior que $20 \mathrm{cam} . / \mathrm{m}^{2}$. O sistema de produção extensivo não foi observado entre as fazendas amostradas.

O Custo Variável Médio representa as despesas com os fatores variáveis empregados na produção por quilo de camarão, o qual é expresso em reais por quilograma $(\mathrm{R} \$ / \mathrm{kg})$. 
Com relação à origem da água de abastecimento dos viveiros, foram definidas as seguintes fontes: rio, considerado como o curso de água natural, que deságua em outro curso, no mar ou lago e; gamboa, definido como zona do leito de um rio onde as águas se apresentam pouco agitadas, semelhante a pequenos lagos.

Para avaliar o nível de adoção das práticas de manejo na fazenda foi utilizado o método de construção de indicadores compostos, seguindo a abordagem proposta por OECD (2008), Saisana (2005) e Nardo et al. (2005).

Seguindo este método, inicialmente, as práticas de manejo foram classificadas em cinco grupos, a saber: (i) manejo de viveiros; (ii) manejo de efluentes; (iii) manejo de sedimentos; (iv) manejo de despesca e; (v) manejo de conservação ambiental. Em seguida, para cada grupo, um indicador foi calculado para refletir seu nível de adoção, denominado de Indicador de adoção de práticas de manejo. Por último, esses indicadores foram agregados em um índice composto, denominado de Índice Global de Adoção de Práticas de Manejo (IGAPM).

De forma genérica, um indicador de adoção de práticas de manejo é obtido por meio do método min-max, que é expresso pela seguinte fórmula (OECD, 2008):

$$
I_{j k}=\frac{\left(V_{j l}-V_{l \text { min }}\right)}{\left(V_{l \max }-V_{l \text { min }}\right.}
$$

onde:

$I_{j k}$ indicador de adoção de práticas do manejo $k$ pela fazenda $j$, sendo $k=1, \ldots, \mathrm{K}$;

$V_{j l}$ : escore atribuído à variável $l$ da fazenda $j$, sendo $l=1, \ldots, \mathrm{n}$;

$V_{l \min }$ : escore mínimo possível assumido pela variável $l$;

$V_{l \max }$ : escore máximo possível assumido pela variável $l$;

$j$ : subscrito que indica a fazenda;

$l$ : subscrito que indica a variável da prática de manejo.

O escore total atribuído a uma categoria de manejo por uma fazenda é obtido pela soma dos escores das práticas adotadas. Para a adoção de uma prática de manejo, atribui-se o escore 1 ; para a não adoção, $0 . \mathrm{O}$ valor do indicador varia no intervalo de 0 a 1 , sendo que 0 significa ausência de adoção e 1 significa adoção de todas as práticas daquele tipo de manejo.

O Índice Global de Adoção de Práticas de Manejo (IGAPM), proposto para refletir o nível global de adoção das práticas na fazenda, é calculado pelo método linear de agregação, em que se assume que as ambiguidades relacionadas aos efeitos de escala são neutralizadas. Matematicamente, o IGAPM é calculado pela seguinte expressão:

$I G G A_{j}-\frac{1}{k}\left(l_{j 1}+l_{j 2}+\ldots+l_{j k}\right) \cdot 100$

onde:

IGAPM: índice da fazenda j:

$I_{j k}$ : indicador $k$ da fazenda $j$;

$j$ : subscrito que indica a fazenda;

$k$ : subscrito que indica o indicador de manejo. 
Os indicadores de manejo e o índice global são calculados para cada uma das fazendas e o total da amostra.

A análise da produtividade média da área cultivada de camarão é feita com base na estimativa de dois modelos: (i) Modelo 1: regressão da produtividade média em relação a todas as variáveis explicativas, exceto o IGAPM; (ii) Modelo 2: regressão da produtividade média com relação a todas as variáveis, exceto os índices de manejo (IMV, IME, IMD e ICA).

\section{RESULTADOS E DISCUSSÃO}

Nesta seção, inicialmente definiu-se o perfil produtivo das fazendas amostradas. Em seguida, descreveu-se o padrão de gestão produtiva e ambiental, com base nas práticas adotadas nas fazendas. posteriormente foram mostrados os resultados das estimativas dos indicadores e índice composto. Por último foram analisados os modelos estimados para identificar os fatores determinantes da produtividade média das fazendas de camarão cultivado.

\section{Perfil produtivo das fazendas}

Em termos médios, as fazendas apresentaram área de 107,1 ha, sendo que a área de viveiros ocupa 48,1 ha. Assim, os viveiros ocupam aproximadamente $45 \%$ da área da propriedade (Tabela 3). Isto indica que, em média, as fazendas dispõem de área para expandir seus cultivos e, assim, aumentar sua produção.

\begin{tabular}{|c|c|c|}
\hline Variável & Média & $\mathrm{DP}^{1}$ \\
\hline \multicolumn{3}{|l|}{ Caracterização da Fazenda } \\
\hline Área da fazenda (ha) & 107,1 & 184,6 \\
\hline Área de viveiros (ha) & 48,1 & 97,3 \\
\hline Produtividade média (kg/ha/ciclo) & $2.500,0$ & $1.525,0$ \\
\hline Densidade de estocagem (cam./ m²) & 33,0 & 15,7 \\
\hline Custo médio operacional de produção ( $\mathrm{R} \$ / \mathrm{kg})$ & 6,9 & 1,9 \\
\hline Sistema de Produção & N. & $\begin{array}{c}\text { Percentual } \\
(\%)\end{array}$ \\
\hline \multicolumn{3}{|l|}{ Sistema de cultivo: } \\
\hline Extensivo & 0 & 0,0 \\
\hline Semi-intensivo & 11 & 18,3 \\
\hline Intensivo & 49 & 61,7 \\
\hline \multicolumn{3}{|l|}{ Assistência técnica existente na fazenda: } \\
\hline Responsável técnico da fazenda & 47 & 78,3 \\
\hline Consultorias periódicas & 32 & 53,3 \\
\hline Técnico em aquicultura & 6 & 10,0 \\
\hline Gestor ambiental & 2 & 3,3 \\
\hline Extensão universitária & 2 & 3,3 \\
\hline Não recebe assistência técnica & 9 & 15,5 \\
\hline
\end{tabular}

Nota: (1) DP significa Desvio Padrão.

Fonte: Elaboração dos autores.

A produtividade média da área cultivada foi de 2,50 t/ha/ciclo, mostrando-se bastante variável, com desvio padrão de $1.525 \mathrm{~kg} / \mathrm{ha} /$ ciclo. O custo médio operacional foi de 
$\mathrm{R} \$ 6,9 / \mathrm{kg}$, com desvio padrão de $\mathrm{R} \$ 1,90 / \mathrm{kg}$. Como será mostrado a seguir, o fato da maioria das fazendas amostradas adotarem o mesmo sistema de cultivo pode ter contribuído para reduzir a amplitude de variação do custo médio operacional.

Rodrigues \& Borba (2013), baseados nos dados do Censo da Carcinicultura 2011 (ABCC, 2013), constataram que, no Ceará, 70\% das fazendas adotavam o sistema semi-intensivo de produção. Na amostra obtida nesse estudo, este sistema de produção foi observado em 11 fazendas (ou 18,3\%), portanto, bem abaixo do percentual observado no Censo. $\mathrm{Na}$ amostra, a média da densidade de estocagem foi de 33 camarões $/ \mathrm{m}^{2}$ e a lâmina de água variou entre 11 a 100 ha, isto devido à predominância do sistema intensivo na amostra.

Ainda segundo a Tabela 3, os produtores indicaram que recorrem à assistência técnica por meio de profissional contratado pela própria fazenda $(78,3 \%)$, consultoria periódica $(53,3 \%)$ e técnico especializado em aquicultura $(10 \%)$. Um percentual elevado de fazendas $(15,5 \%)$ não possui qualquer tipo de assistência técnica no local. Esse resultado sugere que a gestão ambiental mostrou ser uma preocupação secundária, uma vez que apenas 3,3\% das fazendas recorrem a profissionais especializados na área.

\section{Análise das práticas de manejo}

Esta seção discute a adoção das práticas de gestão ambiental nas fazendas de carcinicultura amostradas, cuja apresentação segue a seguinte ordem: (i) manejo de viveiros; (ii) manejo de efluentes; (iii) manejo de despesca; (iv)manejo de sedimentos e; (v) conservação ambiental.

\section{Manejo de viveiros}

As práticas de manejo de viveiros e suas respectivas frequências de adoção pelas fazendas de carcinicultura são apresentadas na Tabela 4.

\begin{tabular}{lcc}
$\begin{array}{l}\text { Tabela } 4 \text { - Frequência absoluta e relativa da adoção de práticas relacionadas ao manejo } \\
\text { de viveiros. }\end{array}$ & N. & $\begin{array}{c}\text { Percentual } \\
\mathbf{( \% )}\end{array}$ \\
\hline Sistema / Práticas & 56 & 93,3 \\
\hline Checagem e limpeza das comportas de drenagem & 47 & 78,3 \\
Monitoramento no entorno da fazenda (pH e salinidade) & 55 & 91,7 \\
Uso de telas filtradoras na entrada e saída de água dos viveiros & 36 & 60,0 \\
Controle do volume de água utilizado na produção & 42 & 70,0 \\
Realização de calagem nos viveiros & 35 & 58,3 \\
Uso de probioticos
\end{tabular}

Fonte: Elaboração dos autores.

Do total da amostra, 93,3\% deles fazia a checagem e limpeza das comportas de drenagem. A ampla adoção dessa prática pelas fazendas pode estar associada aos benefícios que ela proporciona à produção tal como a redução no tempo de despesca que, por sua vez, leva ao aumenta a taxa de sobrevivência do camarão e evita perdas na qualidade do produto.

A grande maioria dos produtores $(91,7 \%)$ também adotou o uso de telas filtradoras na entrada e saída de água dos viveiros. Esta técnica previne a perda de produção por impedir o escape de camarões para fora do viveiro. 
Do total da amostra, 78,3\% das fazendas adotaram o monitoramento do pH e da salinidade no entorno das fazendas. Esperava-se uma maior taxa de adoção dessa prática, uma que vez que esta faz parte do monitoramento ambiental contido no programa de biossegurança das fazendas de camarões marinhos.

O controle do volume de água utilizado na produção foi adotado por $60 \%$ das fazendas amostradas. Isto pode ser explicado pela predominância do sistema de cultivo semi-intensivo nas fazendas, o que torna desnecessário o controle de volume de água, quando se utiliza densidades de estocagem baixa ou média nos cultivos.

A prática de calagem dos viveiros foi adotada por $70 \%$ das fazendas amostradas. A calagem neutraliza a acidez do solo entre os ciclos de produção e aumenta as concentrações de alcalinidade e dureza total da água de cultivo. A realização da calagem está diretamente relacionada à fonte de água de abastecimento dos viveiros.

\section{Manejo de efluentes}

As práticas de manejo de efluentes e suas respectivas frequências de adoção pelas fazendas de carcinicultura são apresentadas na Tabela 5.

Tabela 5 - Frequência absoluta e relativa da adoção de práticas relacionadas ao manejo de efluentes.

\begin{tabular}{lcc}
\hline Sistema/Práticas & N. & $\begin{array}{c}\text { Percentual } \\
\mathbf{( \% )}\end{array}$ \\
\hline Realização de recirculação de água & 18 & 30,0 \\
Despejo dos efluentes nos manguezais & 13 & 21,7 \\
Despejo dos efluentes nas bacias de sedimentação & 32 & 53,3 \\
Despejo dos efluentes na gamboa e/ou rio & 20 & 33,3 \\
Monitoramento e controle do volume total de efluentes da produção & 18 & 30,0 \\
\hline
\end{tabular}

Fonte: Elaboração dos autores.

Um pouco mais da metade das fazendas amostradas (53,3\%) despejava seus efluentes na bacia de sedimentação. Embora a legislação ambiental exija esta prática como um dos requisitos para a obtenção ou renovação da licença ambiental, a taxa de adoção dessa prática não se mostrou elevada, talvez porque mais da metade das fazendas cearense não possui sua licença de operação regularizada.

Do total da amostra, $55 \%$ das fazendas despejavam seus efluentes em rios, gamboas ou manguezais, um percentual ligeiramente maior do que o das fazendas que faziam o manejo adequado dos efluentes, ou seja, despejando os efluentes em bacias de sedimentação.

O manejo dos efluentes dentro da fazenda tem um efeito direto na qualidade da água que é lançada no meio ambiente. Pinheiro et al. (2014) afirmam que as águas de drenagem dos viveiros de produção podem representar um risco potencial de eutrofização nos recursos hídricos receptores.

Aproximadamente um terço dos carcinicultores (30\%) realizava igualmente as práticas de recirculação de água e monitoramento e controle de volume total de efluentes. Apesar de essa prática ter sido instituída pela Resolução do Conselho Nacional de Meio Ambiente (CONAMA) 312/02 como parte dos projetos de carcinicultura, sua adoção efetiva não tem sido observada, talvez devido aos elevados custos de investimento envolvidos na implantação e operação do sistema. 


\section{Manejo de despesca}

As práticas de manejo de despesca e suas respectivas frequências de adoção pelas fazendas de carcinicultura estão apresentadas na Tabela 6.

Tabela 6 - Frequência absoluta e relativa da adoção de práticas relacionadas ao manejo de despesca.

\begin{tabular}{lrc}
\hline Sistema/Práticas & N. & $\begin{array}{c}\text { Percentual } \\
\text { (\%) }\end{array}$ \\
\hline Uso de máquinas de despesca & 3 & 5,0 \\
Despesca manual & 57 & 95,0 \\
Uso de metabissulfito de sódio & 38 & 63,3 \\
Monitoramento do metabissulfito de sódio residual durante a despesca & 27 & 45,0 \\
Uso de equipamentos de proteção individual pelos funcionários/visitantes & 40 & 66,7 \\
\hline
\end{tabular}

Fonte: Elaboração dos autores.

Sobre o método de despesca, constatou-se que apenas três fazendas declararam fazer uso de máquinas na despesca dos viveiros (Tabela 7). A predominância da despesca manual pode ser atribuída à maior disponibilidade de mão de obra e ao valor relativamente baixo da diária do trabalhador rural na zona costeira. ${ }^{6}$

Um número considerável de fazendas declarou fazer uso do metabissulfito de sódio na despesca, tendo sido apontada por $63,3 \%$ dos carcinicultores. O metabissulfito de sódio é um agente oxidante usado para prevenir a formação da melanose (manchas negras ou "black spot") em camarões. Trata-se de um forte agente redutor e compete com a tirosina pelo oxigênio molecular e, após reagir com a água, libera o gás dióxido de enxofre $\left(\mathrm{SO}_{2}\right)$.

O guia intitulado Princípio para Boas Práticas de Manejo (BPM) na engorda de Camarão Marinho no estado do Ceará (Nunes et al., 2005) recomenda uma série de práticas para o manejo correto do metabissulfito, tais como o uso de máscara anti-pó, a provisão de barreiras de contenção e/ou lona encerada na área de manipulação dessa substância e uso de tanques para choque térmico com solo abaulado, a fim de impedir infiltração no solo e vazamento da solução. Sua neutralização deve ser feita através da oxidação do metabissulfito em bisulfato.

$\mathrm{Na}$ amostra, apenas $45 \%$ dos produtores realizava o monitoramento sistemático dos resíduos dessa substância e $66,7 \%$ das fazendas fazia uso de equipamentos de proteção individual durante a despesca.

\section{Manejo de sedimentos}

A Tabela 7 apresenta a distribuição de frequência da adoção das práticas de manejo de sedimentos nas fazendas amostradas.

Tabela 7 - Frequência absoluta e relativa da adoção de práticas relacionadas ao manejo de sedimentos.

\begin{tabular}{lcc}
\hline Sistema / Práticas & N. & $\begin{array}{c}\text { Percentual } \\
\text { (\%) }\end{array}$ \\
\hline Utilização de sistema de tratamento de águas residuais & 14 & 23,3 \\
Realização de manutenção dos canais de drenagem & 39 & 65,0 \\
Utilização de bacias de decantação dos sedimentos & 32 & 53,3 \\
Armazenamento de água de esgotos sanitários e servidas em fossas sépticas & 35 & 58,3 \\
\hline
\end{tabular}

Fonte: Elaboração dos autores.

\footnotetext{
${ }^{6}$ A diária do trabalhador rural é aproximadamente $R \$ 30$, em valor de março de 2015 .
} 
A manutenção dos canais de drenagem foi a prática de manejo de sedimento adotada com maior frequência pelas fazendas (65\%). Esta prática tem o objetivo de evitar problemas de drenagem que possam prolongar o tempo requerido para despesca e a perda de qualidade final do produto.

O armazenamento de água de esgotos sanitários e servidas em fossas sépticas foi adotado por $58,3 \%$ dos produtores. Isto pode ter ocorrido talvez devido aos riscos de contaminação que o manejo inadequado desses efluentes pode ter sobre as pessoas, mananciais e o próprio produto.

Em seguida, aparece a utilização de bacias/lagoas para decantação de sedimentos (53,3\%), prática esta exigida para obtenção da licença ambiental. Por último, tem-se a utilização de sistema de tratamento de águas residuais, com 23,3\%.

\section{Conservação ambiental}

A distribuição de frequência das práticas de conservação ambiental é apresentada na Tabela 8 .

$\begin{aligned} & \text { Tabela } 8 \text { - Frequência absoluta e relativa da adoção de práticas relacionadas à conservação } \\
& \text { ambiental. }\end{aligned}$
\begin{tabular}{lrc} 
Sistema / Práticas & N. & $\begin{array}{c}\text { Percentual } \\
\text { (\%) }\end{array}$ \\
\hline Recuperação das áreas atingidas por canais de captação/drenagem & 25 & 41,7 \\
Realização de monitoramento contínuo dos viveiros & 42 & 70,0 \\
Realização de monitoramento do entorno da fazenda & 37 & 61,7 \\
Recuperação de áreas de manguezais degradados & 9 & 15,0 \\
Monitoramento da fauna e flora da fazenda & 12 & 20,0 \\
\hline
\end{tabular}

Fonte: Elaboração dos autores.

Das cinco práticas de conservação investigadas, duas delas tiveram frequência de adoção relativamente baixa, a saber: recuperação de áreas de manguezais degradados $(15 \%)$ e; monitoramento da fauna e flora (20\%). Isso pode ser atribuído à inexistência de uma política direcionada à recuperação dos manguezais e preservação da fauna e da flora.

Do total da amostra, $41,7 \%$ das fazendas recuperavam as áreas atingidas por canais de captação/drenagem. Apesar de esta prática minimizar o risco de erosão superficial causado pela modificação do fluxo natural da água do estuário ou rio, no geral, a maioria das fazendas não percebia isto como uma ameaça.

O monitoramento dos viveiros e do entorno das fazendas foram aquelas que tiveram maior frequência de adoção, tendo sido indicadas por $70 \%$ e 61,7\% dos carcinicultores, respectivamente. A alta taxa de adoção dessas práticas pode ser atribuída à importância que essas práticas têm para a melhoria do desempenho produtivo do cultivo.

\section{Índice global de adoção de práticas de manejo}

A Tabela 9 apresenta a estatística descritiva dos indicadores de manejo e do índice global de adoção de práticas de manejo para a amostra de fazendas de carcinicultura no estado do Ceará. 
Tabela 9 - Estatística descritiva dos índices dos sistemas de manejo e o Índice Global de Práticas de Manejo das fazendas de carcinicultura.

\begin{tabular}{lcccc}
\hline \multicolumn{1}{c}{ Indicador/Índice } & Mínimo & Máximo & Média & CV $^{\mathbf{1}}$ \\
\hline Indicador de Manejo de Viveiros (IMV) & 0,17 & 1,00 & 0,80 & 0,28 \\
Indicador de Manejo de Efluentes (IME) & 0,00 & 1,00 & 0,40 & 0,83 \\
Indicador de Manejo de Despesca (IMD) & 0,20 & 0,80 & 0,60 & 0,42 \\
Indicador de Manejo de Sedimentos (IMS) & 0,00 & 1,00 & 0,50 & 0,60 \\
Indicador de Conservação Ambiental (ICA) & 0,00 & 1,00 & 0,30 & 0,77 \\
$\begin{array}{l}\text { Índice Global de Adoção de Práticas de } \\
\text { Manejo (IGAPM) }\end{array}$ & 0,17 & 0,90 & 0,50 & 0,02 \\
\hline
\end{tabular}

Nota: (1) CV significa coeficiente de variação.

Fonte: Elaboração dos autores.

O valor estimado para cada um dos indicadores e índice representa a proporção das práticas adotadas pelas fazendas na amostra. Por exemplo, para o IMV, seus valores mínimo e máximo, 0,17 e 1,0, significam que, na amostra, no mínimo 17\% e, no máximo, 100\% das práticas investigadas foram adotadas.

Comparando os indicadores de manejo, o manejo de viveiros (IMV) obteve a maior média $(0,8)$, seguida do manejo de despesca $(0,6)$, manejo de sedimentos $(0,5)$, manejo de efluentes $(0,4)$ e conservação ambiental $(0,3)$. Com base no coeficiente de variação, os manejos de viveiros e despesca apresentaram as menores variâncias. Isto evidencia que os carcinicultores têm dado maior ênfase à adoção de práticas de manejo voltadas para aumentar a eficiência técnica produtiva do que para promover a melhoria da qualidade ambiental na fazenda.

Em termos médios, o IGAPM foi estimado em 0,5, o que significa que as fazendas amostradas adotaram $50 \%$ de todas as boas práticas de manejo investigadas. Porém, na amostra, o pior desempenho foi da fazenda que adotou apenas $17 \%$ das práticas investigadas, enquanto o melhor desempenho foi da fazenda que adotou $91 \%$. O coeficiente de variação do IGAPM foi 0,02 ou $2 \%$, que pode ser considerado relativamente baixo em termos globais.

\section{Análise dos Modelos}

Depois de testar várias formas funcionais (linear, lin-log, log-lin e log-log), a função semi-logarítmica (ou lin-log) foi a que melhor ajustou os dados de produtividade média. Essa função foi utilizada para estimar os modelos 1 e 2, como definidos na seção 3.2.

Os modelos 1 e 2 apresentaram coeficientes de determinação ajustado ( $R^{2}$ Ajustado) de 0,263 e 0,274, significando que os modelos explicaram 26,3 e 27,4\% da variabilidade da produtividade média (Tabela 10). Embora com poder explicativo baixo, o Modelo 2 mostrou desempenho ligeiramente superior do que o Modelo 1, de acordo com as estimativas dos $\mathrm{R}^{2}$ ajustado.

Os testes $\mathrm{F}$ dos dois modelos mostraram significativos ao nível de $1 \%$, o que demonstra que as variáveis explicativas conjuntamente são relevantes para explicar a produtividade média.

Segundo Gujarati (2005), em uma análise de regressão, o objetivo não deve ser apenas obter um $\mathrm{R}^{2}$ elevado, mas obter estimativas confiáveis dos coeficientes de regressão.

Os coeficientes das variáveis explicativas estimados pelos dois modelos mostraram-se equivalentes em termos de significância e sinal. As variáveis significativas ao nível de 
5\% foram "densidade de estocagem" (LNDENEST), "fonte de água de abastecimento dos viveiros" (RIOGAMB) e "tipo de assistência técnica" (TECGEN). As demais variáveis não se mostraram significativas, mesmo aquelas que descrevem a adoção de práticas de manejo. A análise desses coeficientes é feita com base nas estimativas do Modelo 2.

\begin{tabular}{|c|c|c|c|c|}
\hline \multirow{2}{*}{ Variáveis } & \multicolumn{2}{|c|}{ Modelo 1} & \multicolumn{2}{|c|}{ Modelo 2} \\
\hline & Coef. & $P>|t|^{1}$ & Coef. & $P>|t|$ \\
\hline LNAREAFAZ & $86,95^{* * *}$ & 0,731 & 142.25 & 0,553 \\
\hline LNAREAVIV & $-197,91^{* * *}$ & 0,523 & $-304,12$ & 0,323 \\
\hline LNDENEST & $1.115,25^{* * *}$ & 0,026 & $1.153,37^{* *}$ & 0,017 \\
\hline LNCVM & $-188,89^{* * *}$ & 0,802 & $-541,54$ & 0,462 \\
\hline APISAL & $-399,07 * * *$ & 0,316 & $-329,31$ & 0,392 \\
\hline RIOGAMB & $-1.643,70^{* * *}$ & 0,001 & $-1.445,68^{* * *}$ & 0,001 \\
\hline TECGEN & $-1.108,83^{* * *}$ & 0,039 & $-1.054,86^{* * *}$ & 0,041 \\
\hline LNIMV & $706,89^{* * *}$ & 0,245 & $n a^{* * *}$ & - \\
\hline LNIME & $-333,10^{* * *}$ & 0,406 & $n a^{* * *}$ & - \\
\hline LNIMS & $-38,48^{* * *}$ & 0,924 & $n a^{* * *}$ & - \\
\hline LNIMD & $129,24^{* * *}$ & 0,765 & $n a^{* * *}$ & - \\
\hline LNICA & $500,30^{* * *}$ & 0,126 & $n a^{* * *}$ & - \\
\hline LNIGAPM & $n a^{2}$ & - & 691,64 & 0,265 \\
\hline CONS & $2.211,98$ & 0,373 & $2.466,07$ & 0,343 \\
\hline N. obs. & 59 & & 59 & \\
\hline $\mathrm{R}^{2}$ & 0,415 & & 0,374 & \\
\hline $\mathrm{R}^{2}$ Ajustado & 0,263 & & 0,274 & \\
\hline F (sig.) & $2,72^{* * *}$ & & $3,73^{* * *}$ & \\
\hline Prob $>$ F & 0.007 & & 0,002 & \\
\hline
\end{tabular}

Em relação ao sinal dos coeficientes das variáveis significativas, a "densidade de estocagem dos viveiros" (LINDENEST) apresentou uma relação positiva com a produtividade média, ou seja, quanto maior a densidade de estocagem, maior a produtividade média alcançada pela fazenda.

Em termos de valor, o coeficiente expressa que, para cada $1 \%$ de aumento na densidade de estocagem, a produtividade média aumenta, em média, 1.153,37 kg/há/ciclo. Considerando intervalos crescentes de densidade de estocagem, as produtividades médias observadas foram de 1.691, 2.912, 3.002 e $3.333 \mathrm{~kg} / \mathrm{ha} /$ ciclo para os intervalos até $20 \mathrm{cam} / \mathrm{m}^{2}$, 20-40 cam $/ \mathrm{m}^{2}, 40-60 \mathrm{cam} / \mathrm{m}^{2}$ e mais de $60 \mathrm{cam} / \mathrm{m}^{2}$, respectivamente.

Quanto à "fonte de água dos viveiros" (RIOGAMB), as fazendas que abastecem os viveiros com água de rio/gamboa estavam associadas a níveis menores de produtividade média. As fazendas que extraem água do rio/gamboa, em média, possuíam produtividade média $1.445,68 \mathrm{~kg} / \mathrm{ha} /$ ciclo menor do que as fazendas que utilizam água do estuário.

Do total das fazendas amostradas, 73,3\% delas abasteciam seus viveiros com água bombeadas do rio/gamboa e possuíam produtividade média de $2.337,59 \mathrm{~kg} / \mathrm{ha} /$ ciclo, enquanto aquelas que abasteciam seus viveiros com água do estuário alcançavam produtividade média de $3.787,5 \mathrm{~kg} / \mathrm{ha} /$ ciclo, sendo esta $62 \%$ maior do que a primeira. 
Quanto ao tipo de assistência técnica na fazenda (TECGEN), os produtores que recorriam aos técnicos contratados pela própria fazenda possuíam produtividade média inferiores. Em termos de valor, o coeficiente revela que as fazendas que tinham um técnico permanente possuíam produtividade média $1.054,86 \mathrm{~kg} / \mathrm{ha} /$ ciclo, menor do que as fazendas que recorriam a outro tipo de assistência técnica (e.g. consultoria).

As fazendas que possuíam um técnico no local, correspondendo a 78,3\% da amostra, obtinham produtividade média de $2.603,3 \mathrm{~kg} / \mathrm{ha} /$ ciclo, enquanto aquelas que recorriam à assistência técnica periódica obtinham produtividade média de $3.161,5 \mathrm{~kg} / \mathrm{ha} /$ ciclo. Isto pode ser explicado pela maior efetividade do consultor em resolver problemas no âmbito da fazenda, o que resulta em aumento da produtividade.

O coeficiente da área dos viveiros (LNAREAVIV), embora estatisticamente insignificante, apresentou sinal negativo, como era de se esperar, já que uma área maior do viveiro faz declinar a medida de produtividade. O coeficiente da variável que identifica a localização dos viveiros (APISAL) foi insignificante, talvez pela baixa variabilidade da produtividade média entre as categorias dessa variável.

Em ambos os modelos, os indicadores e índice composto da adoção de práticas de manejo não se mostraram significativos, ao nível de 5\%. Segundo o Modelo 1, apesar de estatisticamente insignificante, os coeficientes do LNIMV, LNIMD e LNICA assumiram valores positivos, enquanto os do LNIME e LNIMS obtiveram valores negativos.

Segundo o Modelo 2, o coeficiente do LNIGAPM, embora insignificante, teve efeito marginal positivo sobre a produtividade média das fazendas, ou seja, quanto maior o percentual de práticas adotadas na fazenda, maior será sua produtividade média do camarão cultivado.

\section{CONCLUSÕES}

Os resultados revelaram que, no estado do Ceará, o perfil das fazendas de engorda de camarão é bastante diversificado em virtude da elevada variabilidade observada, em termos de área da fazenda, área de viveiros, densidade de estocagem e produtividade média. Pelo fato de as fazendas adotarem predominantemente o sistema intensivo, o custo médio operacional de produção apresentou baixa amplitude de variação. Quanto às práticas de manejo, as fazendas adotaram com maior frequência aquelas direcionadas à manutenção e operação dos viveiros, e despesca manual e; com menor frequência aquelas voltadas para a gestão ambiental.

Em termos médios, o índice global de adoção de práticas de manejo revelou nível médio de adoção, porém, a adoção das práticas estava distribuída de forma desigual entre as categorias de manejo. As fazendas alcançaram valores maiores para os indicadores mais fortemente associados à eficiência técnica produtiva (manejo dos viveiros, sedimentos e despesca) do que aquelas relacionadas à melhoria da qualidade ambiental (manejo de efluentes e conservação ambiental).

O modelo lin-log (semilogarítmica) foi o que melhor ajustou os dados da amostra. Este modelo mostrou que a produtividade média aumenta com o incremento na densidade de estocagem do viveiro, abastecimento dos viveiros com água do estuário ou ainda recorrendo à assistência técnica periódica (e.g. consultoria). A produtividade média não foi sensível à variação na área da fazenda ou de viveiros, à localização dos viveiros e nem aos indicadores ou índice de adoção de práticas de manejo. Isto sugere que o nível de adoção 
de práticas na fazenda ainda se mostra insuficiente para aumentar a produtividade média da carcinicultura.

Considerando que o índice global de adoção de práticas de manejo na carcinicultura foi de 0,5 , ou seja, as fazendas adotaram $50 \%$ do total das práticas de manejo, pode-se especular que incrementos na produtividade média somente ocorrerão a partir de níveis elevados da adoção das boas práticas.

Para a melhoria da qualidade ambiental na carcinicultura, recomenda-se que sejam realizadas campanhas para aumentar o nível de adoção das boas práticas no estado do Ceará, o que permitirá otimizar a produtividade média da fazenda, assim como proteger o meio ambiente.

\section{REFERÊNCIAS BIBLIOGRÁFICAS}

Alencar, J. R.; Horta Júnior, P. A. \& Celino, J. J. Cultivo de camarão branco Litopenaeus vannamei (Boone, 1931) com a Macroalga Ulva Lacuata Linneaus (Chlorophyta) no tratamento de efluentes em sistema fechado de recirculação. Revista de Biologia e Ciências da Terra Bioterra, Campina Grande, v. 10, n. 1, p.117-137, 2010.

Associação Brasileira de Criadores de Camarão - ABCC. Código de Conduta e de Boas Práticas de Manejo e de Fabricação para uma Carcinicultura Ambientalmente Sustentável e Socialmente Justa, Natal, 2005. Disponível em: <http://abccam. com.br/site/wp-content/ uploads/2011/02/Cdigos_de_Conduta_ABCC_2005.pdf>. Acesso em: 09 set. 2017.

Associação Brasileira de Criadores de Camarão - ABCC. Levantamento da Infraestrutura Produtiva e dos Aspectos Tecnológicos, Econômicos, Sociais e Ambientais da Carcinicultura Marinha no Brasil em 2011, Natal, 2013. Disponível em: <http://abccam.com.br/site/ wpcontent/uploads / 2013/12/LEVANTAMENTO-DA-INFRAESTRUTURAPRODUTIVA.pdf>. Acesso em: 23 maio 2014.

Associação Brasileira de Criadores de Camarão - ABCC. Censo da carcinicultura do litoral norte do estado do Ceará e zonas interioranas adjacentes 2015/2016. Natal: ABCC/ MAPA, 2017.

Asuming-Brempong, S. Land Management Practices and their effects on food crop yields

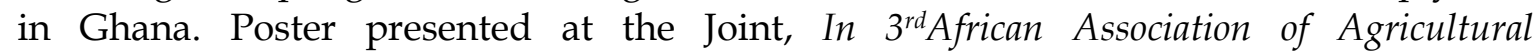
Economists(AAAE) and $48^{\text {th }}$ Agricultural Economists Association of South Africa (AEASA), Conference, Cape Town, South Africa, 2010, p. 19-23.

Brasil. CONAMA n $n^{\circ} 312$, de 10 de outubro de 2002: dispõe sobre o licenciamento ambiental dos empreendimentos de carcinicultura na zona costeira. DOU $n^{\circ} 203$, de 18 de outubro de 2002, Seção 1, p. 60-61.

Feitosa, R. D. Avaliação da Gestão Ambiental da Carcinicultura Marinha no Estado do Ceará: Estudo de Caso. Dissertação (Mestrado em Desenvolvimento e Meio Ambiente) - PRODEMA, Universidade Federal do Ceará, Fortaleza, 2005.

Fonseca, G. B. N. G. Gestão da qualidade no arranjo produtivo da carcinicultura no estado do Rio Grande do Norte. Tese de Doutorado em Ciência Animal, Escola de Veterinária Universidade Federal de Minas Gerais, 135 p., Belo Horizonte, 2005.

Gujarati, D. Econometria Básica. Trad. Maria José Cyhlar Monteiro, 4. ed. Rio de Janeiro: Campus, 2005. 
Instituto Brasileiro de Geografia e Estatística-IBGE. Pesquisa da Pecuária Municipal - 2015. Disponível em: <https://www.ibge.gov.br/estatisticas-novoportal/economicas/ agricultura-e-pecuaria/9107-producao-da-pecuaria-municipal.html $?=\& t=0-q u e-e>$. Acesso em: 27 abr. 2018.

Instituto Brasileiro de Geografia e Estatística-IBGE. Pesquisa da Pecuária Municipal - 2016. Disponível em: <https://www.ibge.gov.br/estatisticas-novoportal/economicas/ agricultura-e-pecuaria/9107-producao-da-pecuaria-municipal.html? =\&t=o-que-e $>$. Acesso em: 27 abr. 2018.

Joventino, F. K. P. A sustentabilidade da carcinicultura no município de Fortim-CE, com ênfase nos aspectos sociais, ambientais e tecnológicos. Revista Pós Ciências Sociais, São Luís, v. 5, n. 9/10, 2008.

Moreno, E. \& Pol, E. Nocionespsicosociales para laintervención y lagestión ambiental. Monografies Socio-Ambientais, v. 14, Publicacions Universitat de Barcelona, Barcelona, 1999. $68 \mathrm{p}$.

Nardo, M.; Saisana, M.; Saltelli, A. \& Tarantola, S. Tools for composite indicators building. European Communication, 2005. Disponível em: <http://publications. jrc.ec.europa.eu/ repository/bitstream/JRC31473/EUR\%2021682\%20EN.pdf>. Acesso em: 13 dez. 2016.

Nunes, A. J. P.; Gesteira, T. C. V.; Oliveira, G. G.; Lima, R. C.; Miranda, P. T. C. \& Madrid, R. M. Princípios para Boas Práticas de Manejo na Engorda de Camarão Marinho no Estado do Ceará. Instituto de Ciências do Mar (Labomar/UFC). Programa de Zoneamento Ecológico Econômico (ZEE) do Estado do Ceará, Fortaleza, Ceará, 2005. 109 p.

Organization for Economic Co-Operation and Development-OECD. Handbook on constructing composite indicators: methodology and user guide. 2008. ISBN 978-92-64-04345-9. Disponível em: <http://www.oecd.org/std/leading-indicators/42495745. pdf>. Acesso em: 13 dez. 2013.

Ormond, J. G. P.; Mello, G. A. T.; Ferreira, P. R. P. \& Lima, C. A. O. A carcinicultura brasileira. BNDS Setorial, Fortaleza, n. 19, p. 91-118, 2004.

Pinheiro, W. M; Aguiar, G. M. B; Silva, L. T; Sobrinho, M. S. \& Guilerme, D. M. R. Monitoramento ambiental das carciniculturas do estado do Ceará no ano de 2013. Universidade Federal do Ceará e Superintendência Estadual do Meio Ambiente, 2014. Disponível em: <http://www.semace.ce.gov.br/wp-content/uploads/2014/11/Banner-SemaceFenacam.pdf> Acesso em: 01 mar. 2015.

Rodrigues, J. \& Borba, M. Carcinicultura Brasileira: estatísticas e revelações. Revista feedEffood, Sorocaba, v. 7, n. 72, 2013. Disponível em: <http://www.revistafeedfood. com. br/pub/curuca/index.jsp?ipg=86345>. Acesso em: 21 fev. 2014.

Saisana, M. (Ed.). State-of-the-art report and composite indicators for knowledge-based economy. Knowledge Economy Indicators - KEY, Workpackage 5, 2005. Disponível em: <https://www.unitrier.de/fileadmin / fb4/projekte/SurveyStatisticsNet/ KEI-WP5-D5.1.pdf>. Acesso em: 13 dez. 2016.

Silva Filho, J. C. L.; Abreu, M. C. S.; Costa, N. B. C.; Cals, B. \& Araújo, N. R. S. Gestão ambiental e social em empresas de carcinicultura: estudo de múltiplos casos no litoral oeste do estado do Ceará. Revista Eletrônica de Administração, Porto Alegre, v. 14, n. 2, p. 472-495, 2008. 
Souza Júnior, J. P. Análise da Eficiência da produção de camarão marinho em cativeiro no estado do Ceará. Dissertação de Mestrado em Economia Rural, Universidade Federal do Ceará, Fortaleza, Ceará, 142 p., 2003.

Tahim, E. F. A carcinicultura e o meio ambiente: o desafio da sustentabilidade. apresentação oralagropecuária, meio-ambiente, e desenvolvimento sustentável. Instituto Centec, Fortaleza, 2006.

Tahim, E. F. \& Araújo Júnior, I. F. A. A carcinicultura do Nordeste brasileiro e sua inserção em cadeias globais de produção: foco nos APLs do Ceará. Revista de Economia e Sociologia Rural, São Paulo, v. 52, n. 3, p. 567-586, jul./set. 2014.

Valenti, W. C. Criação de camarões de água doce. In Anais do Congresso de Zootecnia, 120, Vila Real, Portugal, 2002, Vila Real: Associação Portuguesa dos Engenheiros Zootécnicos, p. 229-237, Vila Real, Portugal, 2002.

Waite, R.; Beveridge, M.; Brummett, R.; Castine, R.; Chaiyawannakarn, N.; Xu, Z., Primavera, J.H.; Peña, L.D. de la; Pettit, P., Belak, J. \& Lcivarwarren, A. Genetic diversity of wild and cultured black tiger shrimp (Penaeus monodon) in the Phillipines using microsatellites. Aquaculture, London, v. 199, n. 1-2, p. 13-40, 2001. 